\title{
On the Mechanism of Free Radical Formation during Browning Reaction of Sugars with Amino Compounds
}

\author{
Tateki HaYASHI and Mitsuo NAMIKI \\ Department of Food Science and Technology, Faculty of Agriculture, \\ Nagoya University, Chikusa-ku, Nagoya 464, Japan
}

Received September 29, 1980

\begin{abstract}
To elucidate the formation mechanism of $N, N^{\prime}$-dialkylpyrazine cation radical during browning reaction of sugars with amino compounds, main products in an early stage of the reaction were determined quantitatively by TLC and GLC. It was shown that the Schiff base, two-carbon fragmental product of sugar, the free radical and deoxyosone were successively produced prior to the browning. Polarographical measurements indicated that the radical formation was induced by the production of some reducing substances in the reaction mixture. These results suggest that the free radical was formed by the reduction of $N, N^{\prime}$-dialkylpyrazinium; a compound, which demonstrated to have a strong activity to browning, might be formed by condensation of twocarbon enaminol followed by oxidation.
\end{abstract}

We have investigated the development of novel free radicals in the early stage of aminocarbonyl reaction of sugars with amino compounds. ${ }^{1)}$ Based on the hyperfine structural analysis of ESR spectra, the radical products were assigned to be $N, N^{\prime}$-dialkylpyrazine cation radicals, ${ }^{2)}$ and it was assumed that each of the alkyl-substituted nitrogens originated from amino compound, and that each of the two-carbon moieties composing the pyrazine ring, from fragmented sugar. We have also confirmed that the two-carbon fragmentation of the sugar molecule occurs prior to the Amadori rearrangement, by isolation and identification of glyoxal derivatives from the ethanolic sugar-amine reaction system. ${ }^{3)}$ The present report deals with the mechanism of the free radical formation in relation to the twocarbon fragmentation, and also with the preliminary study of the role of free radicals in the browning reaction.

\section{EXPERIMENTAL METHODS}

D-Glucosyl- $\beta$-alanine, ${ }^{4)}$ D-glucosyl- $n$-butylamine, ${ }^{\text {S) }} 1-\beta$ alanino-1-deoxy-D-fructose, ${ }^{6)}$ 3-deoxy-D-glucosone ${ }^{73}$ and 1,4-diethylpyrazinium diquaternary salt ${ }^{8)}$ were prepared according to the literatures. Other reagents were of guaranteed grade. Mixtures containing equimolar amounts of a sugar (or a carbonyl compound) and an amino acid (or an amine) were prepared with distilled water or $95 \%$ ethanol, and were heated or refluxed in a water bath.

Amount of the free radical was determined as the relative intensity of its ESR signal to that of the standard $\mathrm{Mn}^{2+}$, by the use of a JES-ME-IX ESR spectrometer with a quartz sample tube for aqueous solutions. The degree of browning was measured by the absorbance at $420 \mathrm{~nm}$. GLC analysis of the the silylated 1- $\beta$-alanino-1deoxyfructose was carried out on a Shimadzu GC-4BMPF with $1.5 \%$ silicone OV-1 column, operated by $2-\mathrm{min}$ initial hold, followed by a $20^{\circ} \mathrm{C} / \mathrm{min}$ temperature program from $90^{\circ} \mathrm{C}$ to $270^{\circ} \mathrm{C}$. The reaction mixture $(0.5 \mathrm{ml})$ with uracil $(0.2 \mathrm{mg})$ added as an internal standard was freezedried and silylated with $1: 1$ BSTFA-acetonitrile $(0.1 \mathrm{ml})$ by heating at $100^{\circ} \mathrm{C}$ for $1 \mathrm{hr}$. 3-Deoxyglucosone was determined by the method described in the previous paper. ${ }^{3)}$ Polarographic analysis with dropping mercury electrode was carried out with Fuso-denki Model 312, in sampled DC and differential pulse (DP) $(20 \mathrm{mV}$ modulation, $5 \mathrm{mV} / \mathrm{s} \mathrm{scan}$ rate) modes. Reaction mixture $(0.1 \mathrm{ml})$ was added to pH 5.5 acetate buffer $(0.2 \mathrm{M}, 5 \mathrm{ml})$, deoxygenated and polarographed. Reducing ability of the reaction mixture was determined by use of the Tillman's reagent. The reaction mixture $(0.01 \mathrm{ml})$ was added to $5 \mathrm{ml}$ of ethanol solution of 2,6-dichlorophenol-indophenol sodium $(5 \mathrm{mg} / \mathrm{liter})$, and after the solution stood for $15 \mathrm{~min}$, the absorbance was measured at $650 \mathrm{~nm}$. The reducing ability was derermined by the decrease of the absorbance from that with blank. 


\section{RESULTS AND DISCUSSION}

Relation of the free radical to the Schiff base, Amadori compound and 3-deoxyglucosone

A generally accepted pathway of an early stage of the browning reaction of sugar with amino compound is initiated with the formation of glycosylamine (a Schiff base), followed by the Amadori rearrangement, and subsequent formation of osones. ${ }^{9}$ ) As has been reported the free radical species is produced at a very early stage of the reaction, prior to the browning. ${ }^{1)}$ In order to examine the radical formation in relation to the formation of these intermediates, an aqueous alkaline solution of D-glucose and $\beta$-alanine was heated in a boiling water bath, and changes in the amount of the free radical, the Amadori compound (1- $\beta$-alanino-1-deoxyfructose), 3-deoxyglucosone, and of the degree of the browning were followed. Figure 1 shows that the free radical develops rapidly prior to or simultaneously with the formation of the Amadori product, and then decreases after about 10 min while the Amadori product still increased. 3Deoxyglucosone gradually increases during these changes. These findings led to the view that the free radical is derived neither from the Amadori product nor naturally from 3deoxyglucosone, and is produced prior to the Amadori rearrangement.

To confirm these relationships, potentiality of these intermediates in giving the free radical was also examined. As shown in Fig. 2, the Amadori product did not provide any free radical by heating alone or with added sugar or amino acid. In contrast to this, glucosyl- $\beta$ alanine alone gave the free radical in a similar extent to that of the glucose- $\beta$-alanine system. In a separate run, the Schiff base, glucosyl- $n$ butylamine alone in ethanol $(1 \mathrm{M})$ also provided the ESR signal in a similar extent to that of glucose- $n$-butylamine system.

It was considered before that the one of the possible ways of the radical formation may be as follows; condensation of the two molecules of the enaminol, such as Amadori compound, produces a 1,4-disubstituted pyrazine deriv-

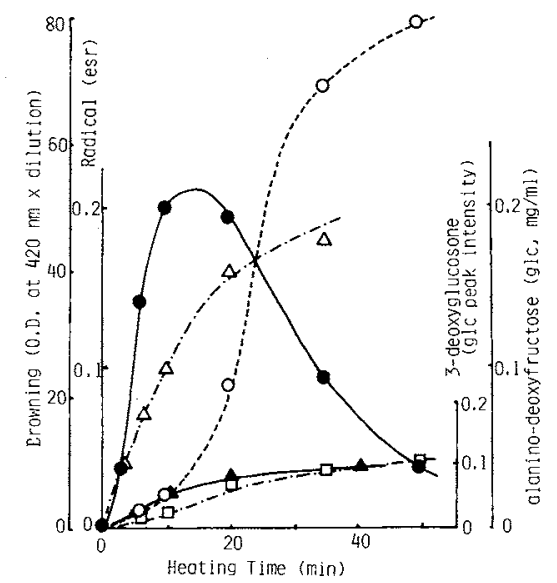

FIG. 1. Formation of Free Radical and Other Products by the Reaction of Glucose with $\beta$-Alanine.

The mixture $(2 \mathrm{M}$ each) with aqueous alkali solution $(0.1 \mathrm{~N} \mathrm{NaOH})$ was heated in a boiling water bath. $\bigcirc$, free radical; $O$, browning; $\triangle$, 1- $\beta$-alanino-1-deoxyfructose; $\square$, 3-deoxyglucosone. Glucose with aqueous alkali solution $(0.1 \mathrm{~N} \mathrm{NaOH})$ was heated as above: browning.

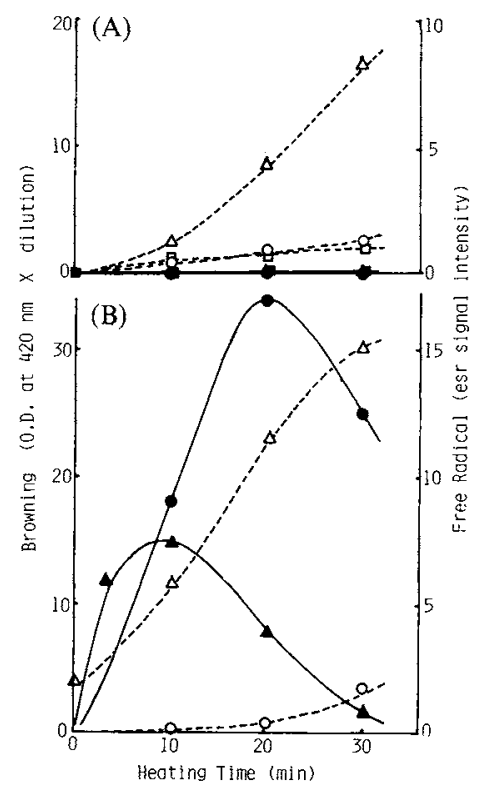

FIG. 2. Development of Free Radicals from Intermediate Products produced by the Reaction of Glucose with $\beta$-Alanine.

Each aqueous solution ( $1 \mathrm{M}$ each) was heated in a boiling water bath. (A) 1- $\beta$-Alanino-1-deoxyfructose: 0 , radical; $\bigcirc$, browning. 1- $\beta$-Alanino-1-deoxyfructose $+\beta$ alanine; $\boldsymbol{\Delta}$, radical; $\Delta$, browning. 1- $\beta$-Alanino-1deoxyfructose + glucose: $\square$, radical, $\square$, browning.

(B) Glucose $+\beta$-alanine: $\bigcirc$, radical; $\bigcirc$, browning. Glucosyl- $\beta$-alanine: $\boldsymbol{\Delta}$, radical; $\triangle$, browning. 
ative carrying two sugar residues at 2,5positions, which loses the sugar moieties by $\mathrm{C}-\mathrm{C}$ fission to give the proposed pyrazine product. The present results, however, revealed that the radical was produced before Amadori rearrangement and eliminated the possibility of the route containing the enaminol of sugar. Then, the remaining possibility is only by the initial fragmentation of sugar to a two-carbon or an enaminol compound.

The formation of the free radical from twocarbon compounds

In the preceding paper, ${ }^{3)}$ glycolaldehydealkylimine, the Schiff base, was proposed as an initial fragmentation product of sugar in the reaction with amine. It was considered to be easily oxidized to glyoxal-monoalkylimine and, subsequently, to give glyoxal-dialkylimine, which is the product isolated from the reaction system. The present results suggest two different ways of the formation of the free radical. The first is the ring formation from enaminol form of glycolaldehydealkylimine, followed by oxidative formation of the free radical, and the second is the cyclization of glyoxal-monoalkylimine to $N, N^{\prime}$-dialkylpyrazinium, which is easily reduced to the free radical. ${ }^{8)}$

The intermediate glycolaldehyde-alkylimine may be formed by the reaction of glycolaldehyde with the amino compound and, the other, glyoxal-monoalkylimine, may be produced from glyoxal and amino compound. Then, the radical formation and browning were compared between these two systems. As shown in Fig. 3, glycolaldehyde system showed far more rapid and stronger radical formation and browning compared with that of glyoxal, indicating that the former is the far more predominantly active intermediate.

The following ESR experiments was made to obtain further evidence of the free radical formation through cyclization of glycolaldehyde-alkylimine or its enaminol. Deuterium-labeled ethanol $\left(\mathrm{C}_{2} \mathrm{H}_{5} \mathrm{OD}\right)$ solution of glycolaldehyde and $t$-butylamine (each $1 \mathrm{M}$ )

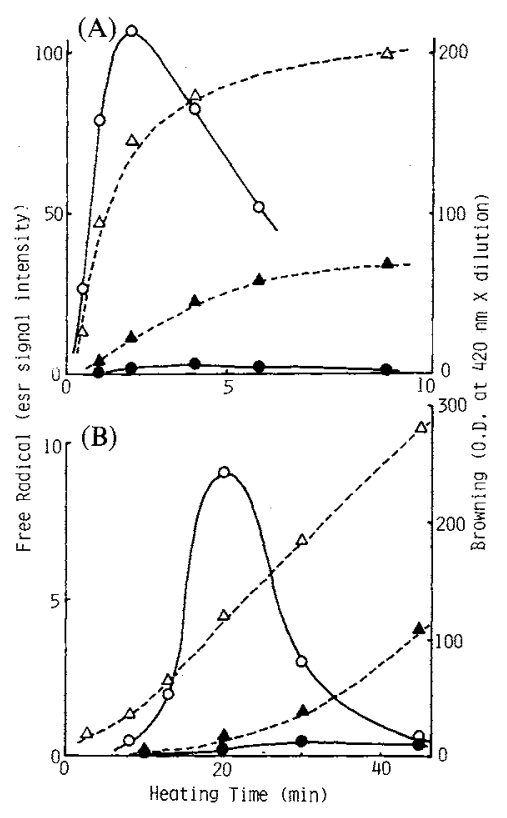

FIG. 3. Formation of Free Radical and Browning by the Reaction of Two-carbon Aldehydes with Amino Compound.

(A) Reaction with $\beta$-alanine ( $1 \mathrm{M}$ each) in water.

(B) reaction with $t$-butylamine $(1 \mathrm{M}$ each) in ethanol. Glycolaldehyde: $O$, radical; $\triangle$, browning. Glyoxal: radical; $\boldsymbol{\Lambda}$, browning.

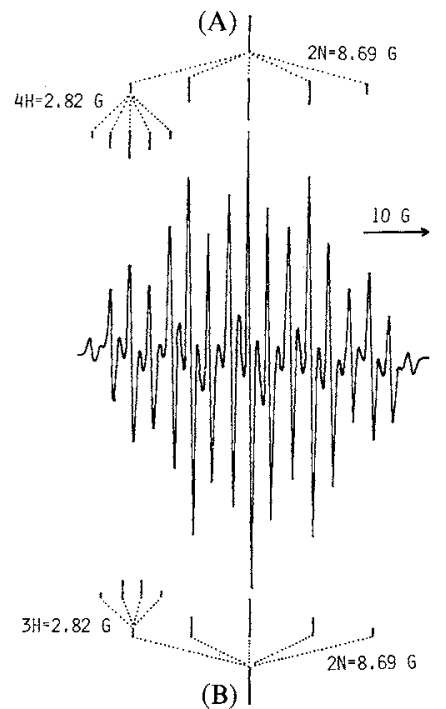

FIG. 4. ESR Spectrum of the Reaction Mixture of Glucose with $t$-Butylamine in Deuteriated Ethanol $\left(\mathrm{C}_{2} \mathrm{H}_{5} \mathrm{OD}\right)$. 
was refluxed for a few min, and the ESR spectrum of the reaction mixture was immediately recorded. The hyperfine structure of the ESR signals (Fig. 4) could be divided into two components $\mathbf{A}$ and $\mathbf{B}$. The component A corresponded to that of the known 1,4-di-tbutylpyrazine radical ${ }^{2)}$ and, $\mathbf{B}$, to the radical in which one of the four ring protons of the pyrazine radical was substituted to deuterium. The substitution might be due to the presence of tautomeric equilibrium between enaminol of glycolaldehyde and its aldehyde type prior to the pyrazine-ring formation. This assumption is supported by the fact that the relative intensity of $\mathbf{B}$ to $\mathbf{A}$ in the ESR spectrum increased significantly when the reaction was made in deuterium oxide in the place of $\mathrm{C}_{2} \mathrm{H}_{5} \mathrm{OD}$.

\section{Formation of reducing substances and their relation to the free radical}

When a reaction mixture of glucose with $t$ butylamine in ethanol refluxed for $50 \mathrm{~min}$ was subjected to sampled DC polarography (Fig. 5 ), there was observed a group of anodic waves consisting of one or two components, which were better resolved by DP polarography. The height of the sampled DC polarographic current at $+0.10 \mathrm{~V}$ plotted against reaction time (Fig. 6) steadily increased with time, after a certain induction time, showing the increase of oxidizable (reducing) substances. The intensity of ESR signal shown simultaneously indicated a similar increase during the reaction, but produces a maximum at about $80 \mathrm{~min}$ and then decreases. The reducing ability of the reaction mixture was also followed by the use of Tillman's reagent (2,6-dichlorophenolindophenol sodium), of which result shown in Fig. 6, is in rough similarity with the polarographic result.

The above results indicate that the reducing ability of the reaction mixture steadily increases with reaction time, after an induction period of about $25 \mathrm{~min}$, and moreover suggest that a certain intermediate compound is formed during the induction period, and is converted by reduction to the radical when the

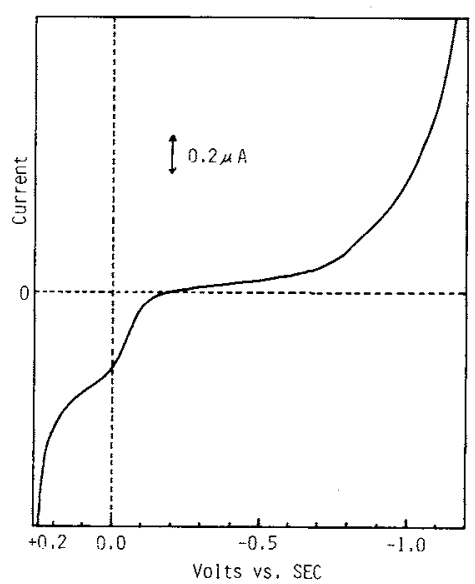

Frg. 5. Sampled DC Polarogram of the Reaction Mixture of Glucose with $t$-Butylamine ( $1 \mathrm{M}$ each) in Ethanol Refluxed for $50 \mathrm{~min}$.

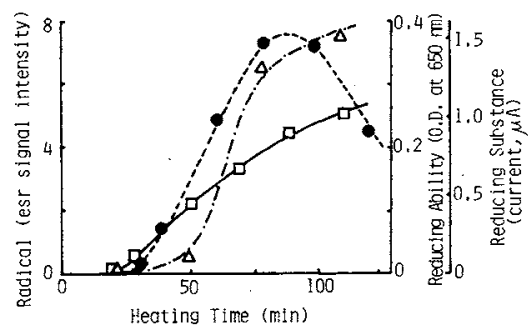

Frg. 6. Formation of Free Radical and the Reducing Substances by the Reaction of Glucose with $t$ Butylamine ( $1 \mathrm{~m}$ each) in Ethanol.

- free radical; $\square$, reducing substances determined by polarography; $\triangle$, reducing ability determined by Tillman's reagent. See text in detail.

reducing ability of the mixture begins to increase. If this is true, addition of certain reductant to the reaction mixture in the induction period, should produce the free radical. The following experiment was carried out to verify this assumption.

\section{Radical precursor in the reaction mixture}

An initial-stage reaction mixture of glucose with $t$-butylamine ( $1 \mathrm{M}$ each) in ethanol was prepared by $15 \mathrm{~min}$ reflux, in which both the free radical and the reducing substances were not yet detected (Fig. 7A and Fig. 6). When aqueous solution of L-ascorbic acid $(0.1 \mathrm{~g} / \mathrm{ml}$, $0.05 \mathrm{ml})$ was added to the mixture $(0.5 \mathrm{ml})$, an 


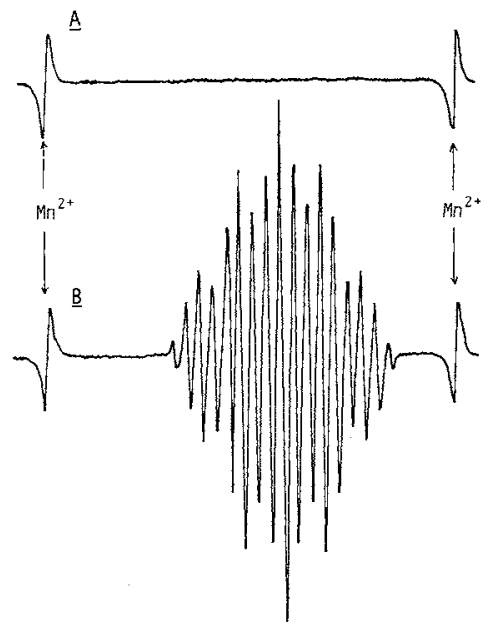

FIG. 7. Appearance of ESR. Spectrum by the Addition of Ascorbic Acid to the Reaction Mixture of Glucose with $t$-Butylamine $(1 \mathrm{M}$ each) in Ethanol Refluxed for 15 min.

(A) Reaction mixture.

(B) Reaction mixture kept at room temperature for $15 \mathrm{~min}$ after the addition of ascorbic acid.

intense ESR signal appeared (Fig. 7B) and increased gradually with time at room temperature. The hyperfine structure of the ESR spectrum was agreed well with that observed in the reaction of glucose with $t$-butylamine. The appearance of free radical by the addition of ascorbic acid to the reaction mixture was also observed in the system of glucose- $n$ butylamine in ethanol $(10 \mathrm{~min})$, and, moreover, though to a lesser degree, in the case of aqueous reaction mixture of glucose- $\beta$-alanine heated for $5 \mathrm{~min}$ in a boiling water bath. Cysteine $\mathrm{HCl}$ acted roughly in the same manner as ascorbic acid, in giving the radical by its addition $(0.1 \mathrm{~g} / \mathrm{ml}, 0.05 \mathrm{ml})$ to the reaction mixture of glucose- $t$-butylamine $(0.5 \mathrm{ml})$ prepared as above. These facts seem to verify that there exist certain intermediate product in the reaction systems, which could easily give the radical product by mild reductants. This intermediate may be tentatively termed as the radical precursor.

The amount of this radical precursor in the reaction mixture of glucose- $t$-butylamine was estimated by the intensity of ESR signal that appeared at $30 \mathrm{~min}$ after the addition of

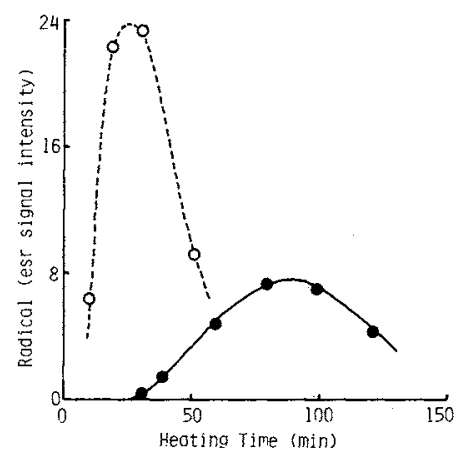

FIG. 8. Formation of the Radical Precursor by the Reaction of Glucose with $t$-Butylamine (1 $\mathrm{M}$ each) in Ethanol.

O, radical precursor; $\mathbf{O}$, free radical. See text in detail.

ascorbic acid. The increase of the ESR signal during the reaction was shown in Fig. 8. The radical precursor was observed at a very early stage of the reaction and increased rapidly within about $25 \mathrm{~min}$, and then decreased along with the development of the free radical. This change in the precursor formation is similar to that observed in the formation of glyoxaldialkylimine as presented in the previous paper, though this two-carbon product is known not to be directly related to the precursor as was mentioned in preceding section.

As to the identity of this precursor, $N, N^{\prime}$ dialkylpyrazinium compounds are the most likely candidate. Its one electron reduction should produce a monocation free radical, and it has been known that diethylpyrazinium compound readily gives the corresponding cation free radical. ${ }^{8)}$ It has been also shown that the reaction of glucose with ethylamine produces the identical free radical species. ${ }^{2)}$ As expected, diethylpyrazinium salt $(1 \mathrm{mg} / 0.5 \mathrm{ml}$ ethanol) gave a large amount of the free radical by the addition of ascorbic acid $(0.1 \mathrm{~g} / \mathrm{ml}, 0.05 \mathrm{ml})$ relative to the control system.

\section{Reaction of 1,4-diethylpyrazinium salt}

As described above, $N, N^{\prime}$-dialkylpyrazinium compound was assigned as the radical precursor. However, formation of dialkyl- 


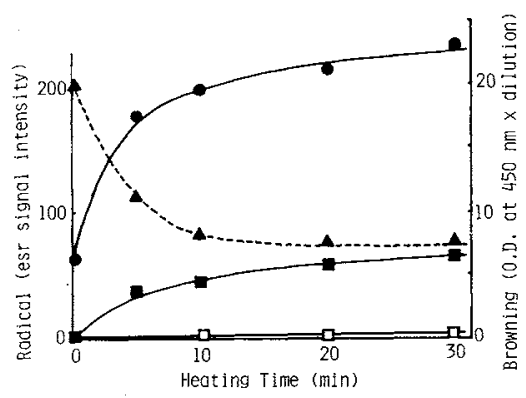

FIG. 9. Changes in Free Radical and Browning of the Solution of 1,4-Diethylpyrazinium Salt and the Mixture of Carbonyl Compounds and Ethylamine.

Diethylpyrazinium in pH 6 phosphate buffer $(50 \mathrm{~mm})$ was heated in boiling water bath: 0 , browning; $\boldsymbol{\Lambda}$, radical. The mixtures of a carbonyl compound and ethylamine ( $50 \mathrm{~mm}$ each) in $\mathrm{pH} 7$ phosphate buffer were heated as above: browning, $\mathbf{a}$, glycolaldehyde + ethylamine; $\square$, glucose + ethylamine.

pyrazinium compound in the amino-carbonyl reaction of sugar with amino compound has not been known. Dialkylpyrazinium compounds are very unstable and will be oxidized easily and decomposed to give ambiguous polymer products. ${ }^{8)}$ The present results suggest a probably important role of this product in the sugar-amino compound browning reaction through the radical formation. The increase of the degree of the browning and change in intensity of the ESR signal by heating of the solution of synthesized diethylpyrazinium salt were followed. As shown in Fig. 9, diethylpyrazinium was very labile and its dissolution in $\mathrm{pH} 6$ buffer solution gave instantaneous browning. Heating enhanced the browning, and its increase was far more rapid as compared with those observed in the sugar-amino reaction mixture. An intense ESR signal was observed immediately after dissolution but decreased rapidly with heating. It was thus shown that this substance is very likely one of the intermediates of the browning reaction.

\section{CONCLUSION}

A pathway on the formation of the novel free radical identified as 1,4-dialkylpyrazine
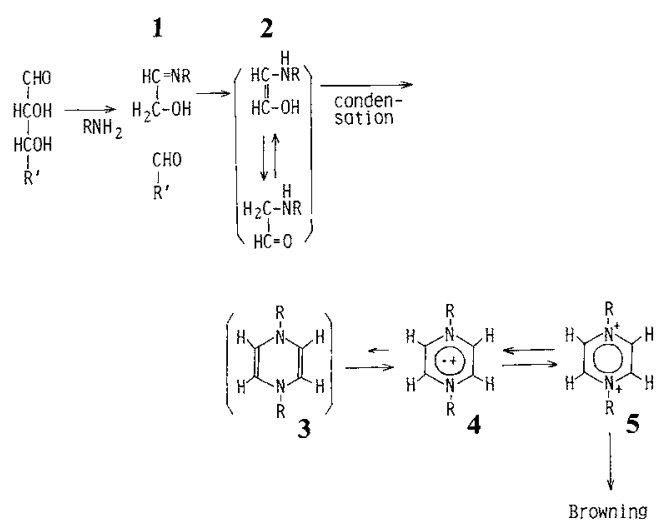

FIG. 10. A Possible Pathway on the Formation of the Free Radical by the Reaction of Sugar with Amino Compound.

cation radical was proposed as in the scheme shown in Fig. 10. Glycolaldehyde-alkylimine (1), the Schiff base, is likely to the initial product at the early stage of this route of browning reaction. ${ }^{3)}$ This product probably undergoes Amadori-type rearrangement and produces enaminol 2, which might exist in a certain tautomeric equilibrium. Condensation of 2 reasonable leads to $N, N^{\prime}$-dialkyldihydropyrazine (3), though this product was not yet detected experimentally, probably due to its unstability. 3 may be easily oxidized to dialkylpyrazinium product (5) via the free radical (4). When the reducing ability of the reaction mixture begins to increase, 5 can give 4 by one-electron reduction. Further study on the relation of this pathway to the known pathway of the general browning reaction of sugar with amino compound is going to be undertaken.

Acknowledgment. Helpful discussions and advice by Dr. Keiichi Tsuji of the Institute of Physical and Chemical Research are greatly appreciated. We thank Miss H. Kato and Mr. T. Hori for technical assistance. This work was supported in part by a grant from the Ministry of Education, Science and Culture of Japan.

\section{REFERENCES}

1) M. Namiki and T. Hayashi, J. Agric. Food Chem., 
23, 487 (1975).

2) T. Hayashi, Y. Ohta and M. Namiki, J. Agric. Food Chem., 25, 1282 (1977).

3) T. Hayashi and M. Namiki, Agric. Biol. Chem., 44, 2575 (1980).

4) G. Weitzel, H. Geyer and A. Fretzdorf, Ber., 90, 1153 (1953).

5) W. Pigman, E. A. Cleveland, D. H. Couch and J. H.
Cleveland, J. Am. Chem. Soc., 73, 1976 (1951).

6) E. F. L. J. Anet, Aust. J. Chem., 10, 193 (1957).

7) H. E. Khadem, D. Horton, M. Meshreki and M. A. Nashed, Carbohyd. Res., 17, 183 (1971).

8) T. J. Curphy and K. S. Prasad, J. Org. Chem., 37, 2259 (1972).

9) H. Kato, Bull. Agric. Chem. Soc., 24, 1 (1960). 